PROCEEDINGS OF THE

AMERICAN MATHEMATICAL SOCIETY

Volume 129, Number 4, Pages 975-981

S 0002-9939(00)05666-5

Article electronically published on December 12, 2000

\title{
GLOBAL EXISTENCE AND BLOWUP OF SOLUTIONS FOR A PARABOLIC EQUATION WITH A GRADIENT TERM
}

\author{
SHAOHUA CHEN
}

(Communicated by David S. Tartakoff)

\begin{abstract}
The author discusses the semilinear parabolic equation $u_{t}=\Delta u+$ $f(u)+g(u)|\nabla u|^{2}$ with $\left.u\right|_{\partial \Omega}=0, u(x, 0)=\phi(x)$. Under suitable assumptions on $f$ and $g$, he proves that, if $0 \leq \phi \leq \lambda \psi$ with $\lambda<1$, then the solutions are global, while if $\phi \geq \lambda \psi$ with $\lambda>1$, then the solutions blow up in a finite time, where $\psi$ is a positive solution of $\Delta \psi+f(\psi)+g(\psi)|\nabla \psi|^{2}=0$, with $\left.\psi\right|_{\partial \Omega}=0$.
\end{abstract}

We study the solutions of the following semilinear parabolic problem:

$$
\begin{array}{cl}
u_{t}=\Delta u+f(u)+g(u)|\nabla u|^{2}, & t>0, x \in \Omega, \\
u(x, t)=0, & t>0, x \in \partial \Omega, \\
u(x, 0)=\phi(x), & x \in \Omega,
\end{array}
$$

where $\Omega \subset R^{n}$ is a bounded domain with smooth boundary $\partial \Omega$.

Kawohl and Peletier $[\mathrm{KP}]$ discussed the blowup behaviors when $f(u)=|u|^{p-1} u$ and $g(u) \equiv c$. Galaktionov [G] obtained exact solutions of $u_{t}-u_{x x}=u^{2}+\left|u_{x}\right|^{2}$ on $R^{+} \times R$. Many authors (see $[\mathrm{KP},[\mathrm{CW}], \underline{\mathrm{S}},[\mathrm{F}]$, and $[\mathrm{D}]$ ) discussed the following problem:

$$
\begin{array}{cl}
u_{t}=\Delta u+|u|^{p-1} u-a|\nabla u|^{q}, & t>0, x \in \Omega, \\
u(x, t)=0, & t>0, x \in \partial \Omega, \\
u(x, 0)=\phi(x), & x \in \Omega .
\end{array}
$$

Existence of global solutions of (2) depends upon the balance between the power of the damping term and that of the source nonlinearity. In one dimension, Deng D] and Fila $[\mathrm{F}$ proved that under suitable assumptions on $p$ and $q$, the solutions of (2) blow up in a finite time if $\phi(x) \geq \psi(x), \forall x \in \Omega$, where $\psi$ is the unique steady state of (2). Deng [D also proved that if $\phi<\psi$, the solutions of (2) approaches zero as $t \rightarrow 0$.

In the case where there is no gradient term, Brezis et al. $[\mathrm{BC}]$ discussed the relations between the existence of global, classical solutions and the existence of weak solutions of the corresponding stationary problem. Under suitable assumptions on $f(u)$, they concluded for (11) with $g(u)=0$ that:

(a) If there exists a global, classical solution of (1), then there exists a weak solution of the stationary problem.

Received by the editors March 2, 1999.

1991 Mathematics Subject Classification. Primary 35K20, 35K55.

Key words and phrases. Parabolic equation, gradient term, global existence, blowup.

(C)2000 American Mathematical Society 
(b) If there is no weak solution of the stationary problem, then for any positive initial value the solution of (1) blows up in finite time.

(c) If there is a weak solution $w$ of the stationary problem, then for any $u_{0} \in$ $L^{\infty}(\Omega)$ with $0 \leq u_{0} \leq w$, the solution of (1) with $u(0)=u_{0}$ is global.

Chen and Derrick CD considered the system

$$
\left(u_{i}\right)_{t}=\Delta u_{i}+f_{i}\left(u_{1}, \ldots, u_{m}\right), \quad t>0, x \in \Omega,
$$

with $\left.u_{i}\right|_{\partial \Omega}=0, u_{i}(x, 0)=\phi_{i}(x)$ and $m \geq 1$. Under suitable assumptions on the nonlinear terms $f_{i}$, they proved that, if $0 \leq \phi_{i} \leq \lambda \psi_{i}$ with $\lambda<1$, then the solutions are global, while if $\phi_{i} \geq \lambda \psi_{i}$ with $\lambda>1$, then the solutions blow up in a finite time, where $\psi_{i}$ are positive solutions of $\Delta \psi_{i}+f_{i}\left(\psi_{1}, \ldots, \psi_{m}\right)=0$, with $\left.\psi_{i}\right|_{\partial \Omega}=0$.

The purpose of this paper is to obtain similar results to those of [CD for the problem (1). We assume that

(i) $g \in C^{1}, g \geq 0$ and $g^{\prime} \geq 0$ for $u>0$ and $u g^{\prime}(u) / g(u)$ is bounded as $u \rightarrow 0^{+}$.

(ii) $\psi$ is a positive solution of $\Delta \psi+f(\psi)+g(\psi)|\nabla \psi|^{2}=0$, with $\left.\psi\right|_{\partial \Omega}=0$.

(iii) $\phi(x) \in C^{2+\beta}(\bar{\Omega})$, for $\beta \in(0,1)$, with $\left.\phi\right|_{\partial \Omega}=0$.

(iv) $f$ is locally Lipschitz continuous, $f(u)=o(u)$ as $u \rightarrow 0^{+}$and $f(u) / u>$ $f(v) / v$ for any $u>v>0$.

(v) $f(u) / u^{\sigma} \geq c_{0}>0$ for some $\sigma>1$ and $u>0$.

Our result is:

Theorem 1. If $0 \leq \phi \leq \lambda \psi$ with $\lambda<1$ and the assumptions (i)-(iv) hold, then the solution $u$ of (1) exists for all $t>0$ and decays exponentially in $t$. If $\phi \geq \lambda \psi$ with $\lambda>1$ and the assumptions (i)-(v) hold, then the solution $u$ of (1) must blow up in a finite time.

Proof. From standard parabolic PDE theory, there exists a unique solution $u(x, t) \in$ $C^{1,0}(\bar{\Omega} \times[0, \tau]) \bigcap C^{2,1}(\bar{\Omega} \times(0, \tau])$ for some $\tau>0$ (see [A1]). By the maximum principle, since $v=\lambda \psi$ is a supersolution of (11) if $\phi \leq \lambda \psi$, we have

$$
0 \leq u \leq \lambda \psi
$$

and since $v=\lambda \psi$ is a subsolution of (II) if $\phi \geq \lambda \psi$, we have

$$
u \geq \lambda \psi \text {. }
$$

We first prove the blowup property. Let $T^{*}$ be the maximal time such that $u(x, t)$ exists. For any number $n$, set

$$
h_{n}(t)=\int_{\Omega} \frac{\psi^{n+2}(x)}{u^{n}(x, t)} d \Omega
$$

for $t \in\left[0, T^{*}\right)$. Differentiating (5), substituting in the equation (1) and integrating by parts (notice that the boundary values are always zero), we have

$$
\begin{aligned}
\frac{d}{d t} h_{n}(t)= & -n \int_{\Omega} \frac{\psi^{n+2}}{u^{n+1}}\left(\Delta u+f(u)+g(u)|\nabla u|^{2}\right) d \Omega \\
= & -n(n+1) \int_{\Omega} \frac{\psi^{n+2}}{u^{n+2}}|\nabla u|^{2} d \Omega+n(n+2) \int_{\Omega} \frac{\psi^{n+1}}{u^{n+1}} \nabla u \nabla \psi d \Omega \\
& -n \int_{\Omega} \frac{\psi^{n+2}}{u^{n+1}} f(u) d \Omega-n \int_{\Omega} \frac{\psi^{n+2}}{u^{n+1}} g(u)|\nabla u|^{2} d \Omega .
\end{aligned}
$$

To simplify (6), we write

$$
\psi^{2}|\nabla u|^{2}=|\psi \nabla u-u \nabla \psi|^{2}+2 u \psi \nabla u \nabla \psi-u^{2}|\nabla \psi|^{2} .
$$


It follows from (6) that

$$
\begin{aligned}
\frac{d}{d t} h_{n}(t)= & -n \int_{\Omega} \frac{\psi^{n}}{u^{n+2}}|\psi \nabla u-u \nabla \psi|^{2}\left[n+1+\left(1-\frac{1}{n}\right) u g(u)\right] d \Omega \\
& -n^{2} \int_{\Omega} \frac{\psi^{n+1}}{u^{n+1}} \nabla u \nabla \psi d \Omega+n(n+1) \int_{\Omega} \frac{\psi^{n}}{u^{n}}|\nabla \psi|^{2} d \Omega \\
& -\int_{\Omega} \frac{\psi^{n+2}}{u^{n+1}} g(u)|\nabla u|^{2} d \Omega+(n-1) \int_{\Omega} \frac{\psi^{n}}{u^{n-1}} g(u)|\nabla \psi|^{2} d \Omega \\
& -2(n-1) \int_{\Omega} \frac{\psi^{n+1}}{u^{n}} g(u) \nabla u \nabla \psi d \Omega-n \int_{\Omega} \frac{\psi^{n+2}}{u^{n+1}} f(u) d \Omega .
\end{aligned}
$$

Using the identity

$$
\begin{array}{r}
-n^{2} \int_{\Omega} \frac{\psi^{n+1}}{u^{n+1}} \nabla u \nabla \psi d \Omega=n \int_{\Omega} \psi^{n+1} \nabla\left(\frac{1}{u^{n}}\right) \nabla \psi d \Omega \\
=-n(n+1) \int_{\Omega} \frac{\psi^{n}}{u^{n}}|\nabla \psi|^{2} d \Omega-n \int_{\Omega} \frac{\psi^{n+1}}{u^{n}} \Delta \psi d \Omega,
\end{array}
$$

and $\Delta \psi=-f(\psi)-g(\psi)|\nabla \psi|^{2}$, we obtain from (7)

$$
\begin{aligned}
\frac{d}{d t} h_{n}(t) \leq & -n \int_{\Omega} \frac{\psi^{n+1}}{u^{n}} \Delta \psi d \Omega-\int_{\Omega} \frac{\psi^{n+2}}{u^{n+1}} g(u)|\nabla u|^{2} d \Omega \\
& -(n+3) \int_{\Omega} \frac{\psi^{n}}{u^{n-1}} g(u)|\nabla \psi|^{2} d \Omega-2 \int_{\Omega} \frac{\psi^{n+1}}{u^{n-1}} g^{\prime}(u) \nabla u \nabla \psi d \Omega \\
& -2 \int_{\Omega} \frac{\psi^{n+1}}{u^{n-1}} g(u) \Delta \psi d \Omega-n \int_{\Omega} \frac{\psi^{n+2}}{u^{n+1}} f(u) d \Omega \\
= & -n \int_{\Omega} \frac{\psi^{n+2}}{u^{n}}\left(\frac{f(u)}{u}-\frac{f(\psi)}{\psi}\right) d \Omega+n \int_{\Omega} \frac{\psi^{n+1}}{u^{n}} g(\psi)|\nabla \psi|^{2} d \Omega \\
& -\int_{\Omega} \frac{\psi^{n}}{u^{n+1}} g(u)\left|\psi \nabla u+\frac{u^{2} g^{\prime}(u)}{g(u)} \nabla \psi\right|^{2} d \Omega \\
& -(n+3) \int_{\Omega} \frac{\psi^{n}}{u^{n-1}} g(u)|\nabla \psi|^{2} d \Omega+2 \int_{\Omega} \frac{\psi^{n+1}}{u^{n-1}} f(\psi) g(u) d \Omega \\
& +2 \int_{\Omega} \frac{\psi^{n+1}}{u^{n-1}} g(\psi) g(u)|\nabla \psi|^{2} d \Omega+\int_{\Omega} \frac{\psi^{n}}{u^{n-3}} \frac{g^{\prime 2}(u)}{g(u)}|\nabla \psi|^{2} d \Omega \\
\leq & -n \int_{\Omega} \frac{\psi^{n}}{u^{n-1}} g(u)|\nabla \psi|^{2}\left(1-\frac{\psi g(\psi)}{u g(u)}-\frac{2}{n} \psi g(\psi)-\frac{u^{2} g^{\prime 2}(u)}{n g^{2}(u)}\right) d \Omega \\
& -n \int_{\Omega} \frac{\psi^{n+1}}{u^{n}} f(\psi)\left(\frac{f(u) / u}{f(\psi) / \psi}-1-\frac{2}{n} u g(u)\right) d \Omega .
\end{aligned}
$$

Using (4) and assumptions (i) and (iv), we can take $n$ sufficiently large that for $t \in\left[0, T^{*}-\delta\right]$ with $\delta>0$

$$
\begin{gathered}
\frac{f(u) / u}{f(\psi) / \psi}-1-\frac{2}{n} u g(u) \geq \epsilon_{n}>0, \\
1-\frac{\psi g(\psi)}{u g(u)}-\frac{2}{n} \psi g(\psi)-\frac{u^{2} g^{\prime 2}(u)}{n g^{2}(u)} \geq 0 .
\end{gathered}
$$


Thus we obtain $h_{n}^{\prime}(t) \leq 0$ or $h_{n}(t) \leq h_{n}(s)$ for $0 \leq s<t \leq T^{*}-\delta$. Taking $n$th roots and letting $n \rightarrow \infty$, we have

$$
\max _{\bar{\Omega}} \frac{\psi(x)}{u(x, t)} \leq \max _{\bar{\Omega}} \frac{\psi(x)}{u(x, s)}
$$

which implies that

$$
v(t)=\sup _{\Omega} \frac{\psi(x)}{u(x, t)}
$$

is decreasing.

To prove that $u(x, t)$ blows up in a finite time we first show that $T^{*}<\infty$. Suppose instead that $T^{*}=\infty$ and $\lim _{t \rightarrow \infty} v(t)=b$. For any $\epsilon>0$, let $\Omega_{\epsilon}=\{x \in$ $\Omega \mid \operatorname{dist}(x, \partial \Omega)<\epsilon\}$. If $b>0$, then $u(x, t)$ is bounded in $\Omega-\Omega_{\epsilon}$. It follows from (8), (10) and assumption (v) that

$$
h_{n}^{\prime}(t) \leq-n \epsilon_{n} \int_{\Omega} \frac{\psi^{n+1}}{u^{n}} f(\psi) d \Omega \leq-n \epsilon^{n} \epsilon_{n} c_{0} \int_{\Omega-\Omega_{\epsilon}} \psi^{1+\sigma} d \Omega
$$

for sufficiently large but fixed $n$, where $\epsilon$ satisfies

$$
\frac{\psi(x)}{u(x, t)} \geq \epsilon
$$

for $x \in \Omega-\Omega_{\epsilon}$ (notice that we temporarily assume $\mathrm{u}$ is bounded). Integrating (11) from 0 to $t$ yields

$$
h_{n}(t)+n \epsilon^{n} \epsilon_{n} c_{0} t \int_{\Omega-\Omega_{\epsilon}} \psi^{1+\sigma} d \Omega \leq h_{n}(0) .
$$

Letting $t \rightarrow \infty$, we get a contradiction. If $b=0$, then for any $N>1$, we have

$$
\frac{\psi(x)}{u\left(x, t_{1}\right)} \leq v\left(t_{1}\right)<\frac{1}{N} \quad \text { or } \quad u\left(x, t_{1}\right) \geq N \psi(x)
$$

for sufficiently large $t_{1}$. If we use this $u\left(x, t_{1}\right)$ as the initial value to solve the problem (11), then the solution must blow up. Since the solution of (1) is unique by the maximum principle, we still have a contradiction. Thus $T^{*}<\infty$. If $u$ is bounded in $\left(0, T^{*}\right)$, then, by [A2], so is $|\nabla u|$, and $u$ can be extended to a time greater than $T^{*}$, which is impossible from the definition of $T^{*}$. Hence $u(x, t)$ blows up as $t \rightarrow T^{*}$.

Now we prove that $u(x, t)$ decays exponentially if $\phi \leq \lambda \psi$ with $\lambda<1$. Similar to the argument above, setting

$$
z_{n}(t)=\int_{\Omega} \frac{u^{n+2}(x, t)}{\psi^{n}(x)} d \Omega
$$


we have

$$
\begin{aligned}
& \frac{d}{d t} z_{n}(t)=(n+2) \int_{\Omega} \frac{u^{n+1}}{\psi^{n}}\left(\Delta u+f(u)+g(u)|\nabla u|^{2}\right) d \Omega \\
& =-(n+2) \int_{\Omega} \frac{u^{n}}{\psi^{n}}|\nabla u|^{2}\left(n+1-\frac{n+3}{n+2} u g(u)\right) d \Omega \\
& +n(n+2) \int_{\Omega} \frac{u^{n+1}}{\psi^{n+1}} \nabla u \nabla \psi d \Omega \\
& -\int_{\Omega} \frac{u^{n+1}}{\psi^{n}} g(u)|\nabla u|^{2} d \Omega+(n+2) \int_{\Omega} \frac{u^{n+1}}{\psi^{n}} f(u) d \Omega \\
& =(n+2) \int_{\Omega} \frac{u^{n+1}}{\psi^{n}} f(u) d \Omega-\int_{\Omega} \frac{u^{n+1}}{\psi^{n}} g(u)|\nabla u|^{2} d \Omega \\
& -(n+2) \int_{\Omega} \frac{u^{n}}{\psi^{n+2}}|\psi \nabla u-u \nabla \psi|^{2}\left(n+1-\frac{n+3}{n+2} u g(u)\right) d \Omega \\
& +(n+2) \int_{\Omega} \frac{u^{n+2}}{\psi^{n+1}} \Delta \psi d \Omega-(n+3) \int_{\Omega} \frac{u^{n+3}}{\psi^{n+2}} g(u)|\nabla \psi|^{2} d \Omega \\
& +2(n+3) \int_{\Omega} \frac{u^{n+2}}{\psi^{n+1}} g(u) \nabla u \nabla \psi d \Omega \\
& =-(n+2) \int_{\Omega} \frac{u^{n+2}}{\psi^{n+1}} f(\psi)\left(1-\frac{f(u) / u}{f(\psi) / \psi}-\frac{2}{n+2} u g(u)\right) d \Omega \\
& -(n+2) \int_{\Omega} \frac{u^{n}}{\psi^{n+2}}|\psi \nabla u-u \nabla \psi|^{2}\left(n+1-\frac{n+3}{n+2} u g(u)\right) d \Omega \\
& -(n+2) \int_{\Omega} \frac{u^{n+2}}{\psi^{n+1}} g(\psi)|\nabla \psi|^{2}\left(1-\frac{u g(u)}{\psi g(\psi)}\right. \\
& \left.-\frac{2}{n+2} u g(u)-\frac{u^{3} g^{\prime 2}(u)}{(n+2) \psi g(\psi) g(u)}\right) d \Omega \\
& -\int_{\Omega} \frac{u^{n+1}}{\psi^{n+2}} g(u)\left|\psi \nabla u+\frac{u^{2} g^{\prime}(u)}{g(u)} \nabla \psi\right|^{2} d \Omega \\
& -3 \int_{\Omega} \frac{u^{n+3}}{\psi^{n+2}} g(u)|\nabla \psi|^{2} d \Omega \text {. }
\end{aligned}
$$

Using (3) and assumption (iv), we can take $n$ sufficiently large such that for sufficiently small $\epsilon_{n}>0$

$$
\begin{gathered}
1-\frac{f(u) / u}{f(\psi) / \psi}-\frac{2}{n+2} u g(u) \geq \epsilon_{n}, \\
n+1-\frac{n+3}{n+2} u g(u) \geq 0, \\
1-\frac{u g(u)}{\psi g(\psi)}-\frac{2}{n+2} u g(u)-\frac{u^{3} g^{\prime 2}(u)}{(n+2) \psi g(\psi) g(u)} \geq 0 .
\end{gathered}
$$


Define $\Omega_{\epsilon}$ as before. Using (12), we obtain

$$
\begin{aligned}
\frac{d}{d t} z_{n}(t) & \leq-(n+2) \epsilon_{n} \int_{\Omega} \frac{u^{n+2}}{\psi^{n+1}} f(\psi) d \Omega \\
& \leq-(n+2) \epsilon_{1} \epsilon_{n} \int_{\Omega-\Omega_{\epsilon}} \frac{u^{n+2}(x, t)}{\psi^{n}(x)} d \Omega \equiv-(n+2) \epsilon_{1} \epsilon_{n} z_{n}^{\epsilon}(t),
\end{aligned}
$$

where $\epsilon_{1}=\min f(\psi) / \psi$ on $\Omega-\Omega_{\epsilon}$. Then

$$
z_{n}^{\epsilon}(t)<z_{n}(t) \leq z_{n}(0)-(n+2) \epsilon_{1} \epsilon_{n} \int_{0}^{t} z_{n}^{\epsilon}(\tau) d \tau,
$$

which implies for fixed $n$ that

$$
\frac{1}{\max \psi^{n}} \int_{\Omega-\Omega_{\epsilon}} u^{n+2}(x, t) d \Omega<z_{n}^{\epsilon}(t) \rightarrow 0, \quad \text { as } \quad t \rightarrow \infty .
$$

Since $u=0$ on the boundary and $u(x, t) \leq \psi(x)$, we have $\int_{\Omega} u^{n+2} d \Omega \rightarrow 0$ as $t \rightarrow \infty$. By $[\mathrm{H}], u(x, t)$ decays exponentially.

Example. For $n=1$, the problem

$$
\begin{aligned}
& u_{t}=u_{x x}+\left(u^{2}+u_{x}^{2}\right) u \quad \text { in }(0, \pi), \\
& u(0, t)=u(\pi, t)=0,
\end{aligned}
$$

with the initial value $\phi(x)=\sin x / \sqrt{1+c}$ has exact solution

$$
u(x, t)=\frac{1}{\sqrt{1+c e^{2 t}}} \sin x .
$$

The corresponding steady state is $\psi(x)=\sin (x)$ in $(0, \pi)$. If $c>0$, then $\phi<\psi$ and the solution is global and exponentially decays. If $-1<c<0$, then $\phi>\psi$ and the solution blows up in a finite time.

Remark. If the problem $\Delta \psi+f(\psi)+a \psi^{p}|\nabla \psi|^{2}=0$, with $\left.\psi\right|_{\partial \Omega}=0$, has two positive solutions, then they must have intersection points by the results of Theorem 1 In fact, assume $\psi_{1}(x)$ and $\psi_{2}(x)$ are solutions with $\psi_{1}(x)>\psi_{2}(x)$ in $\Omega$. We first show that

$$
\sup _{\Omega} \frac{\psi_{2}(x)}{\psi_{1}(x)}<1
$$

If (13) it not true, then there is a point $x_{0} \in \partial \Omega$ such that

$$
1=\lim _{x \rightarrow x_{0}} \frac{\psi_{2}(x)-\psi_{2}\left(x_{0}\right)}{\psi_{1}(x)-\psi_{1}\left(x_{0}\right)}=\frac{\partial \psi_{2}\left(x_{0}\right) / \partial n}{\partial \psi_{1}\left(x_{0}\right) / \partial n},
$$

where $\partial \psi_{1}\left(x_{0}\right) / \partial n<0$ by the strong maximum principle. Let $\psi=\psi_{1}-\psi_{2}$. Then $\psi$ satisfies

$$
\begin{array}{r}
\Delta \psi+\int_{0}^{1}\left[f^{\prime}\left(\psi_{2}+\theta\left(\psi_{1}-\psi_{2}\right)\right)+g^{\prime}\left(\psi_{2}+\theta\left(\psi_{1}-\psi_{2}\right)\right)\left|\nabla \psi_{1}\right|^{2}\right] d \theta \psi \\
+g\left(\psi_{2}\right)\left(\nabla \psi_{1}+\nabla \psi_{2}\right) \nabla \psi=0
\end{array}
$$

$\left.\psi\right|_{\partial \Omega}=0$ and $\partial \psi\left(x_{0}\right) / \partial n=0$. But the strong maximum principle says $\partial \psi(x) / \partial n<$ 0 for all $x \in \partial \Omega$, which is a contradiction. Thus (13) is true. Now we can choose two numbers $\lambda_{1}<1<\lambda_{2}$ and the initial value $\phi(x)$ such that $\lambda_{2} \psi_{2}(x)<\phi(x)<$ $\lambda_{1} \psi_{1}(x)$, leading to a contradiction by Theorem 1] Hence the two solutions must have intersection points in $\Omega$. 


\section{REFERENCES}

[A1] H. Amann, Nonlinear analysis: a collection of papers in honor of Erich Rothe, Academic Press, New York, 1978, 1-29.

[A2] H. Amann, Existence and multiplicity theorems for semi-linear elliptic boundary value problems, Math. Z., 150(1976), 281-295. MR 55:3531

[BC] H. Brezis, T. Cazenave, Y. Martel and A. Ramiandrisoa, Blow up for $u_{t}-\Delta u=g(u)$ revisited, Advances in Diff. Eqs., 1(1996), Vol. 1, 73-90. MR 96i:35063

[CD] S. Chen and W. R. Derrick, Global existence and blow-up of solutions for semilinear parabolic system, Rocky Mountain J. Math. 29(1999), 449-457. CMP 99:16

[CW] M. Chipot and F.B. Weissler, Some blowup results for a nonlinear parabolic equation with a gradient term, SIAM J. Math. Anal., 20(1989), 886-907. MR 90h:35033

[D] K. Deng, Stabilization of solutions of a nonlinear parabolic equation with a gradient term, Math. Z. 216(1994), 147-155. MR 95c:35143

[F] M. Fila, Remarks on blow up for a nonlinear parabolic equation with a gradient term, Proc. Amer. Math. Soc. 111(1991), 795-801. MR 91h:35171

[G] V.A. Galaktionov, On new exact blow-up solutions for nonlinear heat conduction equations with source and applications, Diff. and Int. Eqs., 3(1990), 863-874. MR 91k:35038

$[\mathrm{H}] \quad$ D. Henry, Geometric theory of semilinear parabolic equations, Springer-Verlag, Berlin, Vol. 840, 1981. MR 83j:35084

[KP] B. Kawohl and L.A. Peletier, Observations on blow up and dead cores for nonlinear parabolic equations, Math. Z., 202(1989), 207-217. MR 90k:35035

[P] C.V. Pao, Nonlinear parabolic and elliptic equations, Plenum Press, New York, 1992. MR 94c:35002

[S] P. Souplet, Résultats d'explosion en temps fini pour une équation de la chaleur non linéaire, C. R. Acad. Sc. Paris 321(1995), Série I, 721-726. MR 96h:35093

Department of Mathematics and Statistics, Simon Fraser University, Burnaby, British Columbia, Canada, V5A 1 S6

E-mail address: schend@cs.sfu.ca 